\title{
The Proportion of Radiation Pneumonitis among Lung Cancer Patients in Persahabatan Hospital
}

\author{
Dini Wijayanti ${ }^{1}$, Jamal Zaini ${ }^{1}$, Achmad Hudoyo ${ }^{1}$, Aziza Ghanie ${ }^{2}$, Westi ATW ${ }^{3}$ \\ ${ }^{1}$ Department of Pulmonology and Respiratory Faculty of Medicine, Universitas Indonesia - Persahabatan Hospital, Jakarta \\ 2 Department of Radiology Faculty of Medicine, Universitas Indonesia - Persahabatan Hospital, Jakarta \\ ${ }^{3}$ Department of Radiotherapy, Persahabatan Hospital, Jakarta
}

\section{ARTICLE INFO}

Received : 19 December 2020

Reviewed : 11 February 2020

Accepted : 15 April 2020

\section{Keywords:}

lung cancer, radiation pneumonitis, radiation

\author{
*Corresponding author: \\ Dini Wijayanti \\ Department of Pulmonology and \\ Respiratory Faculty of Medicine, \\ Universitas Indonesia - Persahabatan \\ Hospital, Jakarta \\ dinie30118@yahoo.com
}

\begin{abstract}
Background: Radiation-induced pulmonary toxicity occurs in patients with lung cancer who received irradiation. The objective of this study was to determine the proportion of radiation pneumonitis among lung-cancer patients that were exposed to radiation treatment in Persahabatan Hospital. There is no data about the proportion of radiation pneumonitis in Indonesia.
\end{abstract}

Methods: This was a retrospective study conducted in Persahabatan Hospital during June 2013July 2015 using the medical record of lung-cancer patients who received irradiation.

Results: 33 lung-cancer patients fulfilled the inclusion criteria. Most of them were male (66.7\%), in age $\geq 51$ years (63.6\%), had a smoking history $(75.8 \%)$ with moderate Brinkman Index (60\%), were given irradiation doses of 3,000-4,000 (60.6\%) with irradiation fractions of 10-19 (60.6\%), had no history of chemotherapy (54.5\%), and were diagnosed with adenocarcinoma (66.7\%) and stage-IV cancer (84.84\%). The proportion of radiation pneumonitis based on chest X-ray (CXR) was $39.4 \%$; which consisted of hazy ground-glass opacities, hazy ground-glass opacities, and fibrosis, and only fibrosis. There were significant differences in age, radiation doses, and history of chemotherapy with the proportion of radiation pneumonitis $(p<.05)$.

Conclusions: The proportion of radiation pneumonitis based on CXR was $39.4 \%$. There were significant differences in age, radiation doses, and history of chemotherapy with the proportion of radiation pneumonitis.

\section{INTRODUCTION}

Radiation-induced pulmonary toxicity occurs in patients with lung cancer who received radiation. In lung cancer patients exposed to irradiation, pulmonary toxicity was reported to occur as much as 5-20\% [1]. The progression and spectrum of pulmonary toxicity due to irradiation depended on several factors, including the lung volume which underwent irradiation, the size of the radiation field, the radiation dose, the total amount of radiation given, the time of administration, and individual susceptibility. Radiation can be tolerated if it is given in a small dose, sequentially, and only in one lung. After radiation administration, it was reported that about $40 \%$ of patients had chest X-ray (CXR) abnormalities, and $7 \%$ of them developed symptomatic radiation pneumonitis [1,2].

Radiation pneumonitis images on chest radiographs were rarely found in patients receiving radiation doses below 3,000 cGy. Radiation pneumonitis images on CXR could be observed at doses between 3,000-4,000 cGy and always be seen at doses above 4,000 cGy. The event of radiation pneumonitis was more visible on computed tomography (CT) than in CXR. The radiologic signs shown were in the form of ground-glass opacity and patchy consolidation [2]. Prevention of radiation pneumonitis included the administration of amifostine, captopril, and pentoxifylline [1,2]. There was no data regarding the proportion of radiation pneumonitis in lung-cancer patients exposed to irradiation in Indonesia. This study tried to determine the proportion of radiation pneumonitis in lung cancer patients who received radiation therapy at Persahabatan Hospital.

\section{METHODS}

This was a retrospective study conducted in Persahabatan Hospital from June 2013-July 2015 using the medical record of lung cancer patients who received 
irradiation. The inclusion criteria were lung-cancer patients who received radiation with a minimum dose of 3,000 cGy. The exclusion criteria were incomplete medical record data. The sampling method was done using total sampling of subjects who met the criteria of inclusion and exclusion. Data collection included age, sex, performance status (PS), smoking history, Brinkman Index $(\mathrm{BI})$, radiation dose, radiation fraction, history of chemotherapy, administration of chemotherapy, and CXR 1-month post-radiation. Radiation pneumonitis is an interstitial pulmonary inflammation that can develop in patients receiving thoracic irradiation and can be seen after a 4-to-8-week post-radiation. In this study, the diagnosis of radiation pneumonitis was based on CXR 1-month post-radiation. In Persahabatan Hospital, CXR 1-month post-radiation becomes a routine examination for the lung-cancer patients who received radiation. The evaluation of radiation pneumonitis would be done by review and expertise from a thoracic radiologist on chest radiograph 1-month post-radiation. The data obtained were then analyzed using Chi-square and Fisher test on Statistical Package for Social Science (SPSS) 16. This research was approved by Universitas Indonesia ethical commission number 1083/UN2.F1/ETIK/2015.

\section{RESULTS}

A total of 64 lung cancer patients received irradiation but only 33 subjects had complete medical record data and chest radiograph 1-month post-radiation. Secondary data that met the inclusion criteria of 33 subjects consisted of 11 females and 22 males. The histologic type of lung cancer which obtained radiation were adenocarcinoma and squamous cell carcinoma. Lung cancer patients who received radiation alone were as many as 18 subjects while those who received both radiation and chemotherapy were 15 subjects. The administration of radiation was carried out after chemotherapy or concurrent with chemotherapy.

\section{The proportion of radiation pneumonitis based on CXR}

The diagnosis of radiation pneumonitis in this study was based on CXR in 1-month post-radiation. In lung cancer patients who received irradiation, 13 subjects experienced radiation pneumonitis. The proportion of lung cancer patients who received radiation at Persahabatan Hospital was $39.4 \%$ as seen in Figure $\mathbf{1 .}$

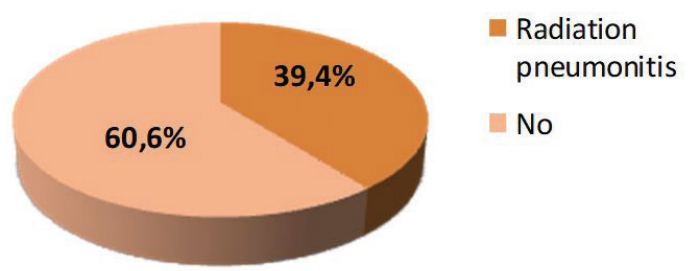

Figure 1. Sample characteristics

Chest radiographs in this study were divided into two categories: those with signs of radiation pneumonitis and no signs of radiation pneumonitis. There were 13 $(39.4 \%)$ CXRs found to have signs of radiation pneumonitis. Those 13 CXRs with radiation pneumonitis consisted of five with the feature of hazy ground-glass opacities, five with both hazy ground-glass opacities and fibrosis, and three with the feature of fibrosis. All of these are illustrated in Figure 2.

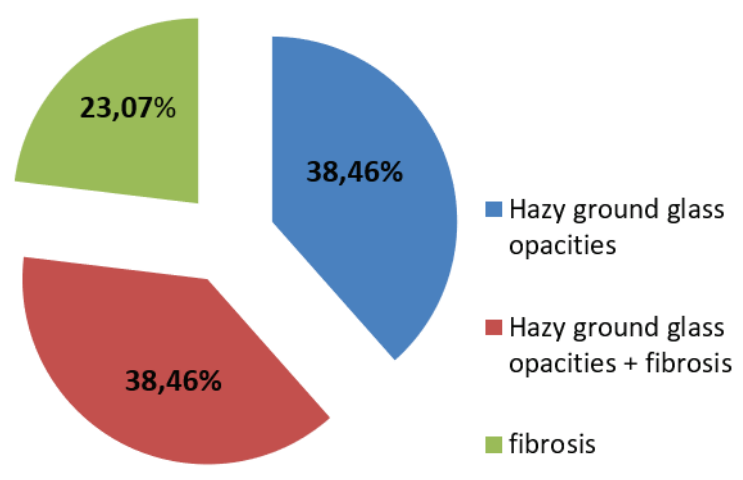

Figure 2. Overview by chest X-ray radiation pneumonitis

\section{The correlation of subject characteristics and radiation pneumonitis}

Age, radiation dose, and history of chemotherapy had significant differences in the event of radiation pneumonitis. The study found a significant difference in the age of lung cancer patients who received radiation with the occurrence of radiation pneumonitis $(P=.043)$. There was also a significant difference between the event of pneumonitis at the radiation doses of $\leq 4,000$ and $>4,000(P=.036)$. Lung-cancer patients who received chemotherapy before irradiation had a significant difference in the event of radiation pneumonitis from those who did not $(P=.027)$. Sex, smoking history, PS, and histologic type did not have significant differences with the occurrence of radiation pneumonitis $(P>.05)$. Age, radiation dose, and history of chemotherapy have a higher risk of radiation pneumonitis. The correlation between characteristics of subjects and radiation pneumonitis can be seen in Table 1 . 
Table 1. Correlation between characteristics of subjects with radiation pneumonitis

\begin{tabular}{|c|c|c|c|}
\hline \multirow{2}{*}{ Variable } & \multicolumn{2}{|c|}{ Radiation pneumonitis } & \multirow{2}{*}{$\mathbf{P}$} \\
\hline & No & Yes & \\
\hline \multicolumn{4}{|l|}{ Age (years) } \\
\hline $30-50$ & 10 & 2 & 0.043 \\
\hline$\geq 51$ & 10 & 11 & \\
\hline \multicolumn{4}{|l|}{ Sex } \\
\hline Male & 12 & 10 & 0.314 \\
\hline Female & 8 & 3 & \\
\hline \multicolumn{4}{|l|}{ Smoking history } \\
\hline Yes & 13 & 12 & 0.074 \\
\hline No & 7 & 1 & \\
\hline \multicolumn{4}{|c|}{ Performance status (PS) } \\
\hline 1 & 6 & 7 & 0.171 \\
\hline 2 & 14 & 6 & \\
\hline \multicolumn{4}{|l|}{ Radiation dose (cGy) } \\
\hline $3000-4000$ & 15 & 5 & 0.036 \\
\hline$>4000$ & 5 & 8 & \\
\hline \multicolumn{4}{|l|}{ Radiation fraction } \\
\hline 10-19 & 14 & 6 & 0.171 \\
\hline 20-30 & 6 & 7 & \\
\hline \multicolumn{4}{|c|}{ History of chemotherapy } \\
\hline Yes & 6 & 9 & 0.027 \\
\hline No & 14 & 4 & \\
\hline \multicolumn{4}{|l|}{ Type of cancer } \\
\hline Adenocarcinoma & 15 & 7 & 0.460 \\
\hline $\begin{array}{l}\text { Squamous cell } \\
\text { carcinoma }\end{array}$ & 5 & 6 & \\
\hline \multicolumn{4}{|l|}{ Stage } \\
\hline III & 3 & 2 & 0.669 \\
\hline IV & 17 & 11 & \\
\hline
\end{tabular}

\section{Subject Characteristics}

The mean age of 13 lung cancer patients who experienced radiation pneumonitis was 58.15 with a dominant age $>50$ years of $69.2 \%$. There were more lung cancer patients with radiation pneumonitis who were males (66.7\%) than females (23.1\%). Twelve patients had a smoking history $(92.3 \%)$ with moderate IB (58.3\%). Lung cancer patients with PS 2 (46.2\%) had more radiation pneumonitis than those with PS 1 . There were 7 patients $(53.8 \%)$ who received doses above 4,000 cGy and radiation fractions of 20 to 30 had radiation pneumonitis. Nine patients with radiation pneumonitis had a history of chemotherapy. Lung cancer patients who developed radiation pneumonitis with the histologic type of adenocarcinoma and stage IV were $53.8 \%$ and $84.6 \%$, respectively. The characteristics of the subjects with lung cancer patients who have radiation pneumonitis are listed in Table 2.
Table 2. Characteristics of subjects with lung cancer who experienced radiation pneumonitis

\begin{tabular}{|c|c|c|}
\hline Variables & $n=13$ & $\%$ \\
\hline Age (years) (mean \pm SD) & $58.15 \pm 9.672$ & \\
\hline $30-50$ & 4 & 30.8 \\
\hline$\geq 51$ & 9 & 69.2 \\
\hline \multicolumn{3}{|l|}{ Sex } \\
\hline Female & 3 & 23.1 \\
\hline Male & 10 & 76.9 \\
\hline \multicolumn{3}{|l|}{ Smoking history } \\
\hline Yes & 12 & 92.3 \\
\hline No & 1 & 7.7 \\
\hline \multicolumn{3}{|l|}{ Brinkman Index (BI) } \\
\hline Mild & 1 & 8.3 \\
\hline Moderate & 7 & 58.3 \\
\hline Severe & 4 & 33.3 \\
\hline \multicolumn{3}{|l|}{ Perfomance Status (PS) } \\
\hline 1 & 7 & 53.8 \\
\hline 2 & 6 & 46.2 \\
\hline 3 & - & \\
\hline 4 & - & \\
\hline Radiation dose (Cgy) (mean \pm SD) & $4723.08 \pm 1.344$ & \\
\hline $3000-4000$ & 6 & 46.2 \\
\hline$>4000$ & 7 & 53.8 \\
\hline Radiation fraction (median, range) & $20(10-30)$ & \\
\hline 10-19 & 6 & 46.2 \\
\hline 20-30 & 7 & 53.8 \\
\hline \multicolumn{3}{|l|}{ History of chemotherapy } \\
\hline Yes & 9 & 69.2 \\
\hline No & 4 & 30.8 \\
\hline \multicolumn{3}{|l|}{ Chemotherapy regimen } \\
\hline Carboplatin + paclitaxel & 5 & 55.55 \\
\hline Carboplatin + etoposide & 4 & 44.45 \\
\hline \multicolumn{3}{|l|}{$\begin{array}{l}\text { Given chemotherapy and } \\
\text { radiation }\end{array}$} \\
\hline Sequentially & 5 & 55.55 \\
\hline Concurrently & 4 & 44.45 \\
\hline \multicolumn{3}{|l|}{ Type of cancer } \\
\hline Adenocarcinoma & 7 & 53.8 \\
\hline Squamous cell carcinoma & 6 & 46.2 \\
\hline \multicolumn{3}{|l|}{ Stage } \\
\hline III & 2 & 15.38 \\
\hline IV & 11 & 84.6 \\
\hline
\end{tabular}

\section{DISCUSSION}

Our study obtained a significant difference in the age of lung cancer patients who received radiation with the occurrence of radiation pneumonitis with a $P=043$. A similar result was obtained by the study from Ivan et al. [3] which pointed out that increasing age would elevate the risk of radiation pneumonitis by 1.7 times 
with $P<.0001$. A study from Youngkyong et al. [4] found that age $<65$ years or $\geq 65$ years did not significantly cause radiation pneumonitis within 6 months $(P=.363)$. The study from Kharofa et al. [5] stated that age $>70$ years increased the risk of radiation pneumonitis with $P=.01$. However, this study did not explain the pathogenesis of the correlation between age and the event of radiation pneumonitis.

Our study found no significant differences between sex and the proportion of radiation pneumonitis $(P=.34)$. This result was following the study from Youngkyong et al. [4] which also found that sex did not affect the event of radiation pneumonitis even though males experienced radiation pneumonitis more often $(P=.224)$ [4]. Ivan et al. [3] stated that there were no significant differences between sex and the event of radiation pneumonitis. The study from Baker et al. [6] revealed that female sex increased the risk of radiation pneumonitis.

There were no differences in smoking history with the occurrence of radiation pneumonitis $(P=.074)$. The different result was found in the study from Ivan et al. [3] which mentioned that smoking in irradiation was a protective factor with an odds ratio (OR) of 0.6 times and a $P=.008$. Patients with smoking history increased the risk by 0.7 times. Smoking history increased the event of radiation pneumonitis as a result of the preexisting lung damage. Youngkyong et al. [4] stated that smoking history was not related to the incidence of radiation pneumonitis $(P=.230)$. Some studies claimed that smoking history could increase the risk of radiation pneumonitis, but active smokers could protect the lung from radiation damage [7].

There were no significant differences in the performance status/PS of lung-cancer patients at the time of radiation with the proportion of radiation pneumonitis $(P=.171)$. Youngkyong et al. [4] found that PS $\leq 1$ or $\geq 2$ was not related to the incidence of radiation pneumonitis $(P=.130)$. The study from Takeyuki et al. [8] stated that PS was not related to the event of radiation pneumonitis. The diagnosis was made in this study using a CXR or thoracic CT scan in 2-week to 1-month post-radiation.

Our study obtained a significant difference between the occurrence of pneumonitis at radiation doses $\leq 4,000$ and $>4,000(P=.036)$. No significant difference was found in the radiation fraction with the event of radiation pneumonitis $(P=.171)$. The radiation dose and fraction influenced the exudation process of protein material to the alveoli which promotes damage in air exchange. The results of this study were similar to the study from Joanne et al. [9] which found a significant difference between the radiation dose in a median of 4,800 cGy compared to $3,750 \mathrm{cGy}$ with the incidence of radiation pneumonitis $(P=.001)$. Joanne et al. [9] stated that the radiation fraction was also significantly different in the event of radiation pneumonitis $(P=.013)$. Youngkyong et al. [4] pointed out that the dose $<7500$ cGy or $\geq 7500$ cGy was not related to the occurrence of radiation pneumonitis with a $P=.165$.

Chemotherapy regimens that were given sequentially and concurrently with radiation consisted of carboplatin and paclitaxel (66.6\%), carboplatin and etoposide $(26.6 \%)$, and gefitinib alone $(1.77 \%)$. The chemotherapy regimens of carboplatin and paclitaxel were administered sequentially while the regimens of both carboplatin and etoposide or gefitinib alone were administered concurrently. The administration of irradiation and chemotherapy generated interferon $\beta$ (IFN $\beta$ ) and inflammatory mediators which caused an increase in cytotoxic radiation in the lung [10]. Lung cancer patients who received chemotherapy before radiation had a significant difference from those without chemotherapy for the incidence of radiation pneumonitis $(P=.027)$. The study from Takeyuki et al. [8] found that there were no significant correlations between chemotherapy and radiation pneumonitis with a $P=.222$. This was due to the number of patients who received chemotherapy, which was less than $50 \%$ of the total study sample. Youngkyong et al. [4] obtained that the history of chemotherapy was not related to the incidence of radiation pneumonitis $(P=.531)$.

The histologic types of lung cancer in this study were divided into two groups namely adenocarcinoma and squamous cell carcinoma. The results of our study were similar to the results of the study from Takeyuki et al. [8] which also stated that there were no significant differences between the histologic types of lung cancer and the prevalence of radiation pneumonitis $(P=.638)$. Youngkyong et al. [4] pointed out that histologic types of lung cancer did not correlate with the incidence of radiation pneumonitis. Following our study, Parashar et al. [11] also stated that there were no significant correlations between the histologic types of lung cancer and the occurrence of radiation pneumonitis.

Our study showed that there were no significant differences between the stage and the event of radiation pneumonitis $(P=.669)$. In our study, the lung-cancer stage was divided into two groups which were III and IV. Youngkyong et al. [4] divided the stage of lung cancer into two groups namely I-IIB and IIIA-IV. The results obtained were that the stage was not related to the incidence of radiation pneumonitis with a $P=0.741$. The study from Takeyuki et al. [8] found that there were no correlations between the stage and the prevalence of radiation pneumonitis $(P=.211)$.

The diagnosis of radiation pneumonitis in our study was based on CXR in 1-month post-radiation. The criteria for chest radiograph was only divided into 2 categories: those with signs of radiation pneumonitis and those without signs of radiation pneumonitis. There were 13 CXRs found to have signs of radiation pneumonitis. Those 13 CXRs with radiation pneumonitis consisted of 
five with the feature of hazy ground-glass opacities, five with both hazy ground-glass opacities and fibrosis, and three with the feature of fibrosis. There is a theory which states that hazy ground glass opacities may disappear without sequelae if the lung damage is still limited. In some cases of severe lung damage, the opacities could develop into fibrosis [12]

The results of this study showed that the proportion of lung cancer patients who developed radiation pneumonitis at Persahabatan Hospital was $39.4 \%$. The radiation technique used was Cobalt 60 (Co60). Youngkyong, et al. [4] stated that the proportion of lung cancer patients who experienced radiation pneumonitis at 1 -month post-radiation was $13.2 \%$. This study determined the proportion of radiation pneumonitis using CXR, thoracic CT scan, and CTCAE. The radiation technique used was different from our study, namely linac-based intensity-modulated radiotherapy. This radiation technique reduced normal lung tissue volume exposure and had a high accuracy which followed breathing patterns so that the radiation effect was reduced.

\section{CONCLUSIONS}

The proportion of radiation pneumonitis based on CXR was $39.4 \%$. There were significant differences in age, radiation doses, and history of chemotherapy with the proportion of radiation pneumonitis.

\section{DECLARATIONS}

\section{Competing of Interest}

The authors declare no competing interest in this study.

\section{Acknowledgment}

The authors thank Dr. Jamal Z Ph.D. and Dr. A Hudoyo, pulmonologist, Drlchksan Ag, radiologist, and Dr. Westi, radiotherapist, for their contribution to this manuscript.

\section{REFERENCES}

1. Sause WT, Gregor A. Treatment of NSCLC: radiotherapy. In:Hansen $\mathrm{HH}$, editor. Textbook of lung cancer. London: Martin Dunitz;2000.p.201-7.

2. Fraser RA. Radiation pneumonitis and fibrosis. In: Johkoh T, Muller NL, Fraser RA, Lee KS, editors. Diseases of the lung: radiologic and pathologic correlations 1sted. Philadelphia: LippincottWilliam\& Wilkins;2003.p.230-7.

3. Ivan R, Soren M. A literature-based meta-analysis of clinical risk factors for the development of radiation-induced pneumonitis. Acta Oncologica. 2012;8:976-9.

4. Youngkyong K, Seong EH, Moonkyoo K, Jinhyun C. Predictive factors for radiation pneumonitis in lung cancer treated with helical tomotherapy. J Kor Can Assoc. 2013;45:295-302.

5. Kharofa J, Gore E. Symptomatic radiation pneumonitis in elderly patients receiving thoracic irradiation. Clin Lung Cancer. 2013;14:283-7.

6. Baker R, Han G, Sarangkasiri S, Demarco M, Turke C. Clinical and dosimetric predictors of radiation pneumonitis in a large series of patients treated with stereotactic body radiation therapy of the lung. Int J Radiat Oncol Biol Phys. 2013;85:190-5.

7. Weytjens R, Erven K, Ruysscher D. Radiation pneumonitis: occurrence, prediction, prevention, and treatment R. Belg J Med Oncol. 2013;7(4):105-10.

8. Takeyuki M, Satoshi T, Kazushige.Risk factors for severe radiation pneumonitis in lung cancer.Jpn J ClinOncol. 1999;29:192-7.

9. Joanne N, Clinton M, Sanjeev S, John P, Frank K, David $P$, et al. Stereotactic body radiotherapy for centrally located early-stage non-small cell lung cancer or lung metastases from the research patient registry. Rad Oncol. 2015;10:113-7.

10. William J, Rubin P, Sandra MD, Dombrowki J. Supportive care in thoracic oncology: chemoradiation pneumonitis and esophagitis. In: Movsas B, Langer CJ, Goldberg M, editors. Controversies in lung cancer 3rded. New York:Marcel Dekker;2001.p.389-405.

11. Federica MD, Luigi M, Glusi IF. Portrait of inflammatory response to ionizing radiation treatment. Journal of Inflammation. 2015;10:12-4.

12. Erasmus JJ, Kara B, Munden R. Iatrogenic lung disease and trauma. In: Mulller, Nestor L, editors. High yield imaging:chest 1 st ed. Philadelphia: Saunders;2009.p.1225-38. 